International Journal of Engineering \& Technology, $7(2.16)(2018) 120-124$
International Journal of Engineering \& Technology
SPC
Website: www.sciencepubco.com/index.php/IJET
Research Paper

\title{
Image Denoising Using Discrete Wavelet Transform : A Theoretical Framework
}

\author{
Praveen Bhargava ${ }^{1}$,Shruti Choubey ${ }^{2}$ Rakesh Kumar Bhujade ${ }^{3}$,Nilesh Jain ${ }^{4}$ \\ 1Ph.D. Research Scholar, CA, Mandsaur University, Mandsaur, Madhya Pradesh, India \\ 2Associate Professor, ECE Department,Sreenidhi Institute of Science \&Technology,Hyderabad,India \\ 3 Assistant Professor, CSE Department, Mandsaur University, Mandsaur, Madhya Pradesh, India \\ 4 Assistant Professor, CA Department, Mandsaur University, Mandsaur, Madhya Pradesh, India \\ *Corresponding author E-mail:praveenbharagava@gmail.com
}

\begin{abstract}
Noise is a random variation in brightness and color in image or simply we can say that unwanted signals are called noise. The noise is mixed with original signal and cause may troubles. Due to the presence of noise, quality of image is reduced and other features like edge sharpness and pattern recognition are badly affected. In image denoising methods to improve the results a hybrid filter is used for better visualization. The hybrid filter is composed with the combination of three filters connected in series. The hybridization has performed much better in case of salt and pepper type of noise and for most of the medical image type, either MRI, CT, SPECT, Ultra Sound. PSNR values show major improvement in comparison of other existing methods. Future, the results obtained from the presented denoising experiments would be tried to be improved further by using this method with other transform domain methods. Finally, the results are concluded that the proposed approach in terms of PSNR, MSE improvement is outperformed.
\end{abstract}

Keywords: Denoising, Noise Reduction, Discrete Wavelet Transform (DWT) etc..

\section{Denoising or Noise Reduction}

Clamor decrease is the way toward expelling commotion from a flag. All chronicle gadgets, both simple and computerized, have characteristics that make them vulnerable to commotion. Clamor can be arbitrary or repetitive sound no soundness, or coherent commotion presented by the gadget's system or preparing calculations.

In electronic account gadgets, a noteworthy type of clamor is murmur caused by irregular electrons that, intensely influenced by warm, stray from their assigned way. These stray electrons impact the voltage of the yield flag and in this manner make perceptible commotion.

On account of photographic film and attractive tape, clamor (both unmistakable and capable of being heard) is acquainted due with the grain structure of the medium. In photographic film, the traverse of crumbs in the photo chooses the film's affectability, more delicate film having greater estimated grains. In alluring tape, the greater the grains of the appealing standard tickles additional disposed the average is to racket.[5]

Toward build up in favor of this, larger zones of image or engaging tape could be utilized to bring down the clamor to a worthy purpose. Numerous clamor lessening calculations tend to harm pretty much flags. The nearby flag and-clamor orthogonalization calculation can be utilized to stay away from the dam-ages to signals.

\section{In Seismic Study}

Starting indicators within seismic information is particularly significant for seismic representation, reversal and elucidation, in this manner enormously enhancing the achievement rate in oil and gas exploration. The valuable flag that is spread in the surrounding irregular commotion is of ten ignored and consequently may cause counterfeit brokenness of seismic occasions and antiques in the last relocated picture. [8]

\section{In Audio}

While using straightforward replicating advancement, they may show a kind of bustle called tape mumble. This is related to the atom size and surface utilized as a piece of the alluring mixturei.e. sprinkled taking place record medium, as well as moreover in the direction of the comparative tape speed over strip starts.

4 sorts of uproar diminishment be present: solo completed pervious copy, solo completed mumble reducing, single-completed surface tumult diminish, and codec or twofold completed systems. Single-completed pre-recording systems, (for instance. Singlecompleted mumble decreasing systems, work to reduce confusion as it happens, including both when the record methodology and furthermore for live convey applications. Single completed surface commotion diminishment, is ap-utilized to the of phonograph trace toward reduce noise of cuts, as well assurconfront non linearity. Twofold completed systems have a pre-emphasis process associated in the midst of recording and after that a de-highlight process associated at play-back.[12]

\section{Compander-Based Noise Reduction Systems:}

The key by and large used sound disturbance de-wrinkle system was made via Ray D. in year 1966. Expected used for virtuoso fissional utilize, form A was an encode/interpret sys-tem in which the plentifulness of frequencies in four gatherings was extended in the midst of recording (encoding), by then de-wrinkled propor- 
tionately in the midst of playback (unraveling). The Dy.form B structure was a solo group system proposed in favor of purchaser things. Into meticulous, while copy silent pieces of a sound banner, regularities more than one kilo $\mathrm{Hz}$ will be bolstered.

This has the impact of accelerating the banner to upheaval extent on tape up to ten sound units looking on the hidden sign volume. Once it absolutely was contend back, the decoder pivoted the methodology, basically reducing the commotion level by up to ten sound unit. The Rudy. B system, where as not as capable as R Dy.

Dbx was a fighting basic commotion diminishment sys-tem made via D.E. Blacker, creator of dbx explores focuses. This is utilize a RMS encode/unwind count with the upheaval slanted high frequencies helped, as well as whole banner energized throughout a 2:1 commander. dbx worked over whole discernable information transmission as well as not in the least like Dolby B was unusable as an open completed system. In any case this might achieve equal to30 $\mathrm{dB}$ of hullabaloo decreasing. while basic record accounts utilize repeat modulation intended for the luminance component, sound approach uproar diminish is pointless.[35]

\section{Dynamic Noise Limiter and Dynamic Noise Reduction:}

DNL is a sound commotion reduction system at first introduced by company Philips during year 1971 utilize on CP. The equipment is also in light of a single chip. It was also framed into dynamic noise diminish by National Semiconductor to lessen tumult levels on long-isolate correspondence. At first sold in 1981, DNR is as regularly as conceivable mixed up for the significantly more average Dolby clatter decreasing system. Regardless, un-like Dolby and dbx Type I and Type II clatter diminish structures, dynamic noise limiter and dynamic noise diminish are playback-simply hail planning systems hat don't need the supply to initial be encoded, and that they are often used in conjunction with numerous kinds of up heavily decreasing.[17]

\section{Supplementary Approaches:}

A below average of calculations effort this point recurrence area utilizing a few direct or non directly channels to enclose nearby attributes as well as are frequently knows as time-recurrence channels. Clamor can accordingly be additionally expelled by utilization of ghastly altering instruments, which effort in this time recurrence area, permitting neighborhood changes without influencing close-by flag vitality.

A different way is to characterize a vibrant edge for channeling commotion, that's gotten from the neighborhood flag, once more concerning a close-by time-recurrence district. Everything beneath the sting are separated, everything over the sting, almost like partials of a voice or "needed clamor", are untouched. The district is usual-ly characterized by the world of the flag fast Frequency, as an oversized portion of the flag vitality to be safe-guarded is assumed concerning it. gift day computerized thousand and movie accounts nevermore have to be compelled to stress over tape murmur so straightforward vogue clamor decrease frameworks square measure a little abundant. In any case, AN intriguing flip is that dither frameworks aircon tally add clamor to a flag to boost its quality.[19]

VII.SOFTWARE PROGRAMS:

Most universally useful voice altering programming will have at least one clamor diminishment capacities (Audacity, Wave Pad, and so forth.). Uncommon reason commotion decrease programming programs incorporate GWC, SCN Reduction, SCP, VR and X-OOM Music Clean.

\section{Inside Images}

Pictures enamored each processed cameras Associate in Nursing regular film cameras can get clamor from an assortment of sources. to boot utilization of those photos can often need that the commotion be (somewhat) exhausted - for tasteful functions as in aesthetic work or promoting, or for affordable functions, as an example, $\mathrm{PC}$ vision.

\section{Types of Clamor}

Salt and pepper clamor parts inside the imager altogether utterly completely different in shading or force from their encompassing picture elements; the characterizing trademark is that the estimation of a boisterous pixel bears no affiliation to the shade of encompassing pixels. For the foremost 0.5 this kind of commotion will merely influence few image pixels. Whenever saw, the image contains boring and white dabs, once the term salt and pepper commotion. Run of the mill sources incorporate bits of fresh within the camera and hot or blemished CCD parts.

In Gaussian clamor, each picture element within the image are remodeled from its distinctive incentive by a (generally) very little total. A bar graph, a plot of the live of twisting of a picture element esteem against the repeat with that it oc-dogs, demonstrates a normal circulation of com-motion. whereas completely different disseminations area unit conceivable, the Gaussian (ordinary) distribution is generally a de-cent model, attributable to as way as attainable hypothesis.

In either case, the clamor at varied pixels are often either corresponded or uncorrelated; as a rule, commotion esteems at varied pixels area unit displayed as being autonomous and indistinguishably disseminated, and henceforward unrelated[4]

\section{Removal \& Tradeoffs}

In choosing a excitement diminish reckoning, one should gauge one or two of segments:

- $\quad$ The open computer power associate degreed time available: a propelled camera should apply confusion diminish during a very little live of a second victimization an retiring introduced electronic equipment, whereas a computer has liberally a lot of power and time.

- Whether surrendering some authentic detail is satisfactory just in case it empowers a lot of upheaval to be re-moved

- The traits of the racket and therefore the detail within the picture, to higher choose those selections.[3]

\section{Chroma and Luminance Noise Separation:}

within evident pictures, the foremost superb spatial repeat detail involves usually of assortments in sparkle (luminance detail) instead of assortments in shade (chroma detail). Since any racket diminishment computation ought to attempt to clear upheaval while not surrendering honest to goodness fact as of the picture caught, 1 threat additional conspicuous failure of fact as of light bustle reducing after that saturation commotion diminish basically in light-weight of the means that almost all scenes have negligible high repeat saturation detail regardless.

\section{Linear Smooth Filter}

Smoothing channels have a bent to cloud a photograph, since pixel management regards that ar essentially higher or below the incorporating neighborhood would "spread" over the zone. By virtue of these darkening, straight channels each currently and so used as items of making ready for clatter diminishes; they're, in any case, oft used because the purpose behind nonlinear confusion decreases channels.[13]

\section{Anisotropic Diffusion}

A different strategy on behalf of evacuating clamor is to advance picture beneath a flatting halfway gap condition just like warmth condition that is termed allotropic dissemination. With a spatially consistent dispersion constant, this can be proportional to the heat 
condition or direct mathematician separating, but with a dissemination constant meant to differentiate edges, the clamor is exhausted while not obscuring the perimeters of the image.[14]

\section{A.Non-Local Filters}

A different approach for evacuating clamor depends on nonneighborhood averaging of the extended variety of pixels during a image. specifically, the live of coefficient for a pel depends on the amount of similitude between very little a bit a trifle to a small degree somewhat slightly a little $\}$ fix targeted on it picture element and therefore the little fix fixated on the picture element being de- clamored.

\section{B. Non-linear Filters}

A middle channel is a case of a non-direct channel and, if appropriately planned, is great at saving picture detail. To run a middle channel:

1. Think every pixel in the picture

2. Type the neighboring pixels into arrange in light of their forces.

3. Replace the first estimation of the pixel with the middle an incentive from the rundown.

Middle and different RCRS channels are great at expelling salt and pepper commotion from a picture, and furthermore cause relatively small obscuring of edges, and thus are regularly utilized as a part of PC vision applications. [17]

\section{Wavelet Transform:}

The primary point of a picture denoising calculation is to accomplish both commotion lessening and highlight protection. In this specific situation, wavelet-based techniques are exceptionally compelling. In the wavelet space, the commotion is consistently spread all through coefficients while the majority of the picture data is gathered in a couple of huge ones. There-fore, the principal wavelet-construct denoising strategies were based with respect to thresholding of detail sub-groups coefficients. Notwithstanding, a large portion of the wavelet thresholding techniques experience the ill effects of the downside that the picked limit may not coordinate the particular circulation of flag and clamor segments at various scales and introductions. [35]

\section{Statistical Methods:}

Measurable techniques for image denoising exist additionally, but they're seldom utilized as they're computationally requesting. For mathematician clamor, one will display the pixels in an exceeding ly grayscale image as auto-typically distributed, wherever each pixel's "actual" grayscale esteem is normally taken over with mean cherish the traditional grayscale estimation of its neighboring pixels and a given distinction.[34]

\section{A. Block-Matching Algorithms:}

A piece coordinating calculation can be connected to gather comparable picture parts into covering macro blocks of indistinguishable size, heaps of comparable full scale squares are then separated together in the change area and each im-age section is at long last reestablished to its unique area utilizing a weighted normal of the covering pixels. [33]

\section{B. Random Field:}

Measurable techniques for picture de-clamoring survive in addition, however they are rarely utilize as that are computationally requesting. For man of science clamor, one can display the picture elements in associate passing G-scale picture as autotypic ally distributed, where every picture element's "actual" grayscale es- teem is nor-mally taken with mean love the standard G-scale estimation of its neighboring pixels and a given distinction.[21]

\section{C.Deep Learning:}

Different profound learning approaches are pro-posed to tackle commotion decrease and such image reclamation undertakings. Profound Image previous is one such system that makes utilization of convolution neural system and is specific therein it needs no earlier making ready info.

\section{D.Software Programs:}

Most broadly useful picture and photograph altering programming will have at least one clamor lessening capacities (middle, obscure, despeckle, and so on.). Universally useful picture and photograph altering programming including clamor diminishment functions incorporate Adb PS, GMP, Photo Impact, P-Shop Pro, H-Filter, as well as Dark table.[29]

\section{Discrete Wavelet Transform (DWT):}

In numerical study and helpful examination, a DWT amendment is any moving ridge amendment that the wavelets area unit discretely tested. Equally like alternative moving ridge changes, a key most well-liked stance it's over Fourier changes is transient determination: it catches each repeat and space information (area in time).The primary discrete wavelet was notional by Hungarian mathematician Alfréd Haar. For associate degree info spoke to by a summing up of numbers, the Haar ripple modification could be thought of to match up input esteems, golf shot away the excellence and spending the entire. This procedure is rehashed recursively, mixing up the wholes to demonstrate the subsequent scale that prompts contrasts and a final total.[10]

\section{A.The Dual-Tree Complex Wavelet Transform (DCWT):}

The double hierarchy complicated ripple amendment (CWT) may be a moderately late improvement to the distinct ripple amendment (DWT), with essential additional properties: it's al-most move invariant and directionally specific in 2 and better measurements. It accomplishes this with a redundancy issue of simply munificently less than the undedicated DWT. The threedimensional (M-D) double tree CWT is no separable but depends on a computationally effective, distinguishable channel bank (FB). Different types DWT change incorporate the un-wrecked WT change (someplace down-examining is precluded), the Newland change (someplace an ortho ordinary premise of wavelets is framed from appropriately built best cap channels in recurrence space). Wavelet bundle changes are likewise identified with the discrete wavelet change. Complex wavelet change is another frame.,[35]

\section{B.Properties}

The Haardiscrete wavelet delineates the alluring characteristics of WT all in all. To start with, it can be per-shaped in the particular tasks; second, it catches not just a thought of the recurrence substance of the contribution, by analyzing it at various scales, yet in addition fleeting substance, that is circumstances at which these frequencies happen. Consolidated, these two properties influence the Fast wavelet to change (FWT) an other option to the traditional quick Fourier change (FFT).

Because of the variation in directors within the channel bank, the separate wavelet is not time-invariant but truly very sensitive to the arrangement of the flag in time. to handle the time-fluctuating issue of moving ridge changes, Mallat and Zhong projected another calculation for moving ridge representation of a flag, that is invariant to time shifts. As indicated by this calculation that is understood as a TI-DWT simply the size parameter is examined on the two arrangement and also the moving ridge modification is patterned for every purpose in time.[29] 


\section{Applications}

The distinct rippling modification features a large range of uses in science, designing, arithmetic and computer code engineering. Mainly remarkably, it's utilized for flag secret writing, to talk to a distinct flag in an exceedingly additional redundant form, oftentimes as a preconditioning for data compression. Down to earth applications can likewise be found in flag preparing of increasing speeds for walk examination, picture handling, in computerized interchanges and numerous others.

This is demonstrated the DWT change is effectively actualized the same as simple channel stock in medical flag preparing in support of outline of small control leaders plus also in ultra WB remote correspondences.[17]

\section{Example in Image Processing:}

Wavelets are regularly used to denoise two dimensional signals, for example, pictures. The accompanying illustration gives three stages to expel undesirable white Gaussian clamor from the uproarious picture appeared. Matlab was utilized to import and channel the picture.

The initial step is to pick a wavelet compose, and a level $\mathrm{N}$ of deterioration. For this situation biorthogonal 3.5 wavelets were picked with a level $\mathrm{N}$ of 10 . Biorthogonal wavelets are normally utilized as a part of picture preparing to identify and channel white Gaussian clamor, because of their high differentiation of neighboring pixel power esteems. Utilizing this wavelets a wavelet change is performed on the two dimensional picture.

Following the decay of the picture record, the subsequent stage is to decide edge esteems for each level from 1 to N. Birgé Massmart technique is a genuinely regular method for choosing these edges. Utilizing this procedure singular limits are made for $\mathrm{N}=10$ levels. Applying these edges are most of the genuine channel ing of the flag.

The last advance is to recreate the picture from the modified levels. This is expert utilizing an opposite wavelet change. The subsequent picture, with white Gaussian commotion expelled is appeared underneath the first picture. While separating any type of information it is imperative to measure the flag to-clamor proportion of the outcome. For this situation, the SNR of the boisterous picture in contrast with the first was $30.4958 \%$, and the SNR of the denoised picture is $32.5525 \%$. The subsequent change of the wavelet sifting is a SNR pick up of $2.0567 \%$.

It is critical to take note of that picking different wavelets, le-vels, and thresholding methodologies can bring about various kinds of sifting. In this illustration, white Gaussian clamor was been evacuated. In spite of the fact that, with various thresholding, it could simply have been increased.[34]

\section{Comparison with Fourier Transform:}

To speak to the refinements and comparable qualities among the DWT change with the DFT change, think about the discrete wavelet and discrete Fourier for going with progression: $(1,0,0,0)$, a unit inspiration.

\section{Preparatory perceptions include:}

particularly, center gauge contrasts. Starting repeat space point of view, a superior evaluate, yet starting point zone the point of viewis impediments - this demonstrates below shoot individual qualities is negative, however the primary course of action is positive wherever - as well as sound, some place at the correct face having value, not at all like in the wavelet change.

On the other hand, the Fourier estimation adequately evil spirit stratus a zenith, and all concentrations are within their correct regard, however all concentrations have screw up. The Trough calculation, by separate, puts a best on missing partly, however hasn't best on fundamental position, along with remembering that is accurately acceptable on behalf of a vast part of the characteristics (reflecting territory), it has an error of a fix a motivating force for exchange regards. This speaks to the sorts of trade offs between these progressions, and how in a couple of respects the
DWT gives perfect direct, particularly for the showing of vagabonds.

\section{References}

[1] Chen, Yangkang; Fomel, Sergey (November-December 2015). "Random noise attenuation using local signal-and-noise orthogonalization". Geophysics.

[2] Chen, Yangkang; Fomel, Sergey; Sun, Junzhe (2016). "Seismic imaging of incomplete data and simultaneous-source data using least-squares reverse time migration with shaping regularization". Geophysics.

[3] Chen, Yangkang; Yuan, Jiang; Zu, Shaohuan; Qu, Shan; Gan, Shuwei (2015). "Seismic imaging of simultaneous-source data using constrained least-squares reverse time migration". Journal of Applied Geophysics.

[4] Shruti Bhargava* and Ajay Somkuwar , "Estimation of Noise Removal Techniques in Medical Imaging Data-A Review", Dknmu, Newai MANIT Bhopal, Journal of Medical Imaging and Health Informatics Vol. 6, 1-10, 2016.

[5] Shruti Bhargava and S.P.V. Subbarao, "Evaluation of Different Noise Removal Filterization Techniques in Imaging", Sreenidhi Institute of Science \&Technology,Hyderabad, International Journal of Pure and Applied Mathematics Volume 117 No. 21 2017, 605-612.

[6] Shruti bhargava, Ajay Somkuwar, Denoising Mri Images Using Wavelet Based Bivariate Shrinkage Method, CCET Journal of Science \& Engineering Education Vol. -1, Page-55-61, Year2016

[7] Chen, Yangkang; Chen, Hanming; Xiang, Kui; Chen, Xiaohong (2017). "Geological structure guided well log interpolation for high-fidelity full waveform inversion". Geophysical Journal International.

[8] Gan, Shuwei; Wang, Shoudong; Chen, Yangkang; Qu, Shan; Zu, Shaohuan (2016). "Velocity analysis of simultaneous-source data using high-resolution semblance - coping with the strong noise". Geophysical Journal International.

[9] Chen, Yangkang (2017). "Probing the subsurface karst features using time-frequency decomposition". Interpretation.

[10] Huang, Weilin; Wang, Runqiu; Chen, Yangkang; Li, Huijian; Gan, Shuwei (2016). "Damped multichannel singular spectrum analysis for 3D random noise attenuation". Geophysics.

[11] Chen, Yangkang (2016). "Dip-separated structural filtering using seislet transform and adaptive empirical mode decomposition based dip filter". Geophysical Journal International.

[12] Chen, Yangkang; Ma, Jianwei; Fomel, Sergey (2016). "Doublesparsity dictionary for seismic noise attenuation". Geophysics.

[13] Chen, Yangkang (2017). "Fast dictionary learning for noise attenuation of multidimensional seismic data". Geophysical Journal International.

[14] R., C. (1965). "KompanderverbessertMagnettonkopie". radio mentor (in German). 1965 (4): 301-303.

[15] Burwen, Richard S. (February 1971). "A Dynamic Noise Filter". Journal of The Audio Engineering Society. 19 (1).

[16] Burwen, Richard S. (June 1971). "110 dB Dynamic Range For Tape" (PDF), North American Publishing Company: 49-50.

[17] Shruti Bhargava, Abhishek Choubey, Estimation of Different Thersholding Techniques for Signal Denoising, International Journal of Electronics Communication and Computer Engineering Volume 8, Issue 3, ISSN (Online):pp.153-155.

[18] Burwen, Richard S. (December 1971). "Design of a Noise Eliminator System". Journal of The Audio Engineering Society. 906911.

[19] www.radiomuseum.org/r/gyar_mk42mk_4.html

[20] Hoffman, Frank W. (2004). Encyclopedia of Recorded Sound. 1 (revised ed.). Taylor \& Francis.

[21] Nidhi Upadhyay Shruti Bhargava, , removal of noise in medical imaging data using modified decision based adaptive weighted algorithem, International Journal of Computer Applications , Volume 84 - No 13, December 2013

[22] Gunyo, Ed. "Evolution of the Riviera - 1983 the 20th Anniversary". Riviera Owners Association. (NB. Originally published in The Riview, Vol. 21, No. 6, September/October 2005.)

[23] Boashash, B., ed. (2003). Time-Frequency Signal Analysis and Processing - A Comprehensive Reference. Oxford: Elsevier Science. ISBN 0-08-044335-4.

[24] Shruti Bhargava, A Study on Various Speckle Noise Diminution Techniques, International Journal of Recent Development in Engineering and Technology, ISSN 2347-6435, Volume 6, Issue 2, February 2017 
[25] Boashash, B. (April 1992). "Estimating and Interpreting the Instantaneous Frequency of a Signal-Part I: Fundamentals". Proceedings of the IEEE. 80 (4): 519-538.

[26] Liu, Puyin; Li, Hongxing (2004). Fuzzy Neural Network Theory and Application. World Scientific. ISBN 981-238-786-2.

[27] Forouzanfar, M.; Abrishami-Moghaddam, H.; Ghadimi, S. (July 2008). "Locally adaptive multiscale Bayesian method for image denoising based on bivariate normal inverse Gaussian distributions". International Journal of Wavelets, Multiresolution and Information Processing. 6 (4): 653-664.

[28] Mallat, S. (1998). A Wavelet Tour of Signals Processing. London: Academic Press.

[29] Praveen Bhargava, Shruti Choubey, Rakesh Kumar Bhujade Nilesh Jain, Review Article: A Review Literature on Image Denoising using Discrete Wavelet Transform and Filters, International Journal Of Scientific Progress And Research (Ijspr) Issn: 2349-4689 Issue 120, Volume 42, Number 03, December 2017.

[30] Besag, Julian (1986). "On the Statistical Analysis of Dirty Pictures". Journal of the Royal Statistical Society. Series B (Methodological). 48 (3): 259-302.

[31] Dabov, Kostadin; Foi, Alessandro; Katkovnik, Vladimir; Egiazarian, Karen (16 July 2007). "Image denoising by sparse 3D transform-domain collaborative filtering". IEEE Transactions on Image Processing. 16 (8): 2080-2095.

[32] Schmidt, Uwe; Roth, Stefan (2014). Shrinkage Fields for Effective Image Restoration (PDF). Computer Vision and Pattern Recognition (CVPR), 2014 IEEE Conference on. Colombus, OH, USA: IEEE. ISBN 978-1-4799-5118-5.

[33] Shruti Bhargava, Ajay Somkuwar "Evaluation of Noise Exclusion of Medical Images using Hybridization of Partical Swarm Optimization and Bivariate Shrinkage Methods" International Journal of Electrical and Computer Engineering (IJECE) Vol. 5 No. 3, June 2015, pp. 421 428 ISSN: 2088-8708

[34] Shruti Bhargava, Ajay Somkuwar "Hybrid Filters based Denoising of Medical Images using Adaptive Wavelet Thresholding Algorithm" International Journal of Computer Applications ,Volume 83 - No 3, December 2013.

[35] Shruti Bhargava, Dr. Ajay Somkuwar,noise assessment in medical imaging data, journal of medical imaging \& health informatics ,Volume 6, Number 4, 875-884 August ,2016 